\title{
AUGUSTUSBILD IM RHETORISCHEN WERK VON SENECA RHETOR
}

\begin{abstract}
Summary: In this study, I analyze the literary picture of the emperor Augustus as it is depicted in the rhetorical works of Seneca the Elder. Based on both direct and indirect references in Seneca's collection of the examples of rhetorical tools published under the heading Oratorum et rhetorum sententiae, divisiones, colores, which is better known as Controversiae and Suasoriae, I call into question the usually accepted idea that Seneca admired Augustus for his respect for freedom of speech.

Augustus is mostly ( 6 out of 9 times) mentioned in a private or a schooling environment which is reflected in the stylized and idealized portrait of a learned, brisk, and witty, but also of a very perceptive man, who can fairly be described as "a master of bon mot and a man with a big heart". Four times he is, in these anecdotes, but out of the anecdotic frame, referred to as a publicly active man. Although Augustus as princeps makes the impression of a noble, clement, and admirable man, the tension and fear his power arouses in orators starts to penetrate his idealized depiction. In the remaining references, he is inadvertently and furtively criticized. The criticism concerns the individuals close to Augustus who assist him in performing his duties, especially their low origin, as these were often freedmen and other careerists of low social rank. Another issue, for which Augustus is criticized, are the unforgettable injustices committed during the Second Triumvirate. Seneca's indirect commentaries concerning this matter are very exasperated and wrathful, which suggests that he perceived Augustus' time as the period of the loss of democracy and endangered law. These prove that Seneca the Elder coped with the new situation only unwillingly and with difficulties. The pessimism present in his works is not a common place, but a reflection of the real situation Romans had to face.
\end{abstract}

Key words: Seneca Maior, Augustus, declamations, literary picture, freedom of speech

Das historische Bild vom ersten princeps des Römischen Imperiums Augustus wird dank stets entstehender Studien immer genauer und umfangreicher. Der Bestandteil dieses Bildes ist auch die allmähliche Verwandlung der Stellung des Augustus in der Gesellschaft, die durch die Berichte von Geschichtsschreibern wie auch die legislativen Schritte des Senats und weiterer römischer Ämter ordentlich dokumentiert ist. Die Linie der historischen Belege wird notwendigerweise durch die literarischen Belege ergänzt, die dem aufmerksamen Leser einen Blick „,unter den Mantel“ der offiziellen Erklärungen und Verehrungen ermöglicht. Manche von ihnen beteiligen sich 
mit unterschiedlichem Engagement und Eigenwillen an der Unterstützung der Politik des Schicksalsretters des römischen Friedens - im Klartext werden sie zum Bestandteil der Propaganda. Neben den augusteischen Dichtern wird aufgrund einiger Erwähnungen zu den Augustusunterstützern und seinem Bewunderern auch Seneca Rhetor gezählt, wie die zwar mehr als hundert Jahre alte, aber bisher hochgeschätzte ${ }^{1}$ Dissertation von Thomas Stanley Simonds ${ }^{2}$ belegt, laut welcher Seneca ,in complete sympathy with Augustus whom he terms a ,clementissimus vir,' and praises for allowing to a certain extant freedom of speech," steht. Ähnlich auch der viel spätere Beitrag von Miriam Griffin „The Elder Seneca and Spain“, ${ }^{3}$ dessen Autorin feststellt, dass Seneca „reveals a deep admiration for Augustus, under whom libertas (in the sense of free speech) flourished." Dieser Beitrag soll überprüfen, inwiefern diese verdächtig eindeutige Auffassung von Senecas Beziehung zu Augustus stimmt. Um dieses Ziel zu erreichen, müssen auch diejenigen Stellen bestimmt und interpretiert werden, wo Seneca die Situation im Rom unter der Regierung von Augustus indirekt kommentiert.

Seneca der Ältere oder Seneca Rhetor (um ihn von seinem bekannteren Sohn, dem berühmten Philosophen, zu unterscheiden) stammte aus einer wohlhabenden ritterlichen Familie aus dem hispanischen Corduba, d.h. gehörte den angesehensten Kreisen im römischen Imperium an. ${ }^{4}$ Sein ganzes Leben verbrachte er zwischen Corduba und Rom, höchstwahrscheinlich sein Familienvermögen verwaltend. Soweit wir wissen, ist er nicht in die Ämterlaufbahn eingestiegen. Er ist noch am Ende der Republik zur Welt gekommen, wie er selbst erwähnt im Zusammenhang mit der vereitelten Möglichkeit, den berühmtesten Redner Cicero zu hören. Er räumte ein, dass er Cicero noch erlebt hat, dass er aber wegen des Bürgerkriegs kaum nach Rom fahren konnte.

Sen. Rhet. Contr. 1 praef. 11: Omnes autem magni in eloquentia nominis excepto Cicerone videor audisse; ne Ciceronem quidem aetas mihi eripuerat, sed bellorum civilium furor, qui tunc orbem totum pervagabatur, intra coloniam meam me continuit: alioqui in illo atriolo, in quo duos grandes praetextatos ait secum declamasse, ${ }^{5}$ potui adesse, illudque ingenium quod solum populus Romanus par imperio suo habuit, cognoscere

${ }^{1}$ Vgl. SusSman, L.: The Elder Seneca and Declamation since 1900. In ANRW II 32.1 (1984) $557-577$, hier 559 .

${ }^{2}$ SimONDS, TH. ST.: The Themes Treated by the Elder Seneca. Diss. Univ. John Hopkins, Baltimore 1896, 44.

${ }^{3}$ GRIFFIN, M.: The Elder Seneca and Spain. JRS 62 (1972) 1-19, hier 13 f.

${ }^{4}$ Vgl. FAIRWEATHER, J.: The Elder Seneca and Declamation. In ANRW II 32.1 (1984) 514-556, hier 518 f., und besonders GRIFFIN (Anm. 3) 1-19, die in ihrem Beitrag die Wurzeln des Seneca-Stammes sowie auch die Stellung, die die vornehmen hispanischen Familien im römischen Reich innehatten, ausführlich untersucht.

${ }^{5}$ Es handelt sich um Pansa und Hirtius, consules designati für das Jahr 43 v. Chr. (FAIRWEATHER [Anm. 4] 517). Hirtius und Pansa waren gemeinsam mit Cicero in Puteoli 44 v. Chr. und haben ihn, wie er selbst anderswo erwähnt (Cic. Ad Att. 14. 12), zum Deklamieren gezwungen. (Vgl. SCHÖNBERGER, O. SCHÖNBERGER, E. [Transl.]: Lucius Annaeus Seneca der Ältere. Sentenzen, Einteilungen, Färbungen von Rednern und Redenlehrern. Würzburg 2004, 309, die einen inkorrekten Verweis machen; und auch GRIFFIN [Anm. 3] 5, die von rhetorischen Lektionen, „teaching sessions“, schreibt.) 
et, quod vulgo aliquando dici solet, sed in illo proprie debet, potui vivam vocem audire.

Im Text handelt es sich um das Jahr 44 v. Chr. Man kann also vermuten, Seneca ist gegen das Jahr 55 v. Chr. geboren. Was seinen Tod betrifft, können wir den Zeitraum sicher nach dem Jahr $34 \mathrm{n}$. Chr. setzen, in dem das in seiner Schrift letzte datierte Ereignis passiert ist, nämlich Mamercus Aemilius Scaurus Selbstmord begangen hat (Suas. 2. 2). Aus Senecas Erwähnungen der unter Tiberius verbotenen Autoren, vor allem Cremutius Cordus (Suas. 6. 19-23; seine Historien wurden im Jahr 25 n. Chr. verbrannt), lässt sich weiter ableiten, dass Seneca sein Werk erst nach Tiberius' Tod, unter Kaiser Caligula, veröffentlicht hat ${ }^{6}$ (falls das Werk zur Veröffentlichung noch während seines Lebens bestimmt wurde ${ }^{7}$ ). Im Jahr 41 n. Chr., als sein Sohn, der Philosoph Seneca, verbannt wurde, war der Vater allerdings schon tot (siehe Sen. Phil. Cons. Helv. 2.4 f.).

Als hochgebildeter Laie pflegte Seneca sein otium auch der literarischen Tätigkeit zu widmen. Ganz verloren ist sein historisches Opus, mit dem er angeblich besonders in den letzten Jahren seines Lebens beschäftigt war (Sen. Phil. Frg. 98 Haase $^{8}$ ). In einer anderen Schrift, Oratorum et rhetorum sententiae, divisiones, colores, die sich zwar nicht ganz, aber immerhin erhalten hat, wollte Seneca seinen Söhnen einen umfangreichen Bericht über die Rhetorik in der Zeit von Augustus geben. In zehn Büchern von Controversiae und ein von Suasoriae (die Schrift ist unter diesen beiden Namen bekannt geworden und wird so hier weiter erwähnt) sind zahlreiche Beispiele der unterschiedlichsten rednerischen Mittel von meist augusteischen Rednern zum Nutzen seiner Söhne gesammelt, thematisch geordnet und kommentiert. Seneca vermittelt dadurch den Söhnen, genau wie sie sich gewünscht haben, ${ }^{9}$ ein sehr lebhaftes Bild der Verwandlung der Rhetorik aus einer praktischen Fähigkeit des Forums in eine scholastische, sogar akademische Disziplin. ${ }^{10}$ Gleichzeitig erfasst er, zum Teil

${ }^{6}$ Siehe GRIFFIN (Anm. 3) 4.

${ }^{7}$ FAIRWEATHER (Anm. 4) 517, N. 6. Die Bestimmung zur Veröffentlichung geht jedoch klar hervor aus Contr. 1 praef. 10.

${ }^{8}$ Sen. Phil. Frg. 98 Haase: quisquis legisset eius historias ab initio bellorum civilium, unde primum veritas retro abiit, paene usque ad mortis suae diem, magni aestimaret scire, quibus natus est parentibus ille, qui res Roma〈nas... ... diem 〈perductas〉.

${ }^{9}$ Seneca erwähnt diesen Beweggrund zum Schreiben - den Wunsch seiner Söhne - mehrmals (Sen. Rhet. Contr. 1 praef. 1: Exigitis rem magis iucundam mihi quam facilem; iubetis enim quid de his declamatoribus sentiam, qui in aetatem meam inciderunt, indicare et si qua memoriae meae nondum elapsa sunt ab illis dicta colligere, ut, quamvis notitiae vestrae subducti sint, tamen non credatis tantum de illis sed et iudicetis. Ibid. 7 praef. 1: Instatis mihi cotidie de Albucio. Ibid. 9 praef. 1: Iam videbar promissum meum implesse; circumspiciebam tamen, num quid me prateriret. Ultro Votieni Montani mentionem intulistis, ...). Im zehnten Buch äußert er sogar schon seine Empörung (Ibid. 10 praef. 1: Quod ultra mihi molesti sitis, non est: interrogate, si qua vultis, et sinite me ab istis iuvenilibus studiis ad senectutem meam reverti. Fatebor vobis, iam res taedio est), will allerdings seine Aufgabe vollenden.

${ }^{10}$ Diese Verwandlung wird dabei als ein wichtiger Teil des literarischen Niedergangs interpretiert, der mit der Kaiserzeit eingetreten sein solle (siehe vor allem WILLIAMS, G.: Change and Decline: Roman Literature in the Early Empire. Berkeley - Los Angeles - London 1978, am deutlichsten 2-5), wobei Seneca als die Person beschrieben ist, die die Diskussion über Niedergang der Rhetorik im Prinzipat eröffnet hat (siehe z.B. Sussman, L. A.: The Elder Seneca's Discussion of the Decline of Roman 
absichtlich, zum Teil unbewusst, die Verwandlung der in der republikanischen Tradition verankerten römischen Gesellschaft zur Gesellschaft, die immer sichtbarer und handfester ihre Rechte und Privilegien in die Hände des einzelnen, höchsten Bürgers, des Prinzeps Augustus, delegiert.

Senecas Beziehung zu Augustus und seiner Regierung lässt sich aus direkten sowie auch indirekten Äußerungen erkennen. Und, wie man vermuten kann, das offizielle Bild unterscheidet sich von dem, das man im Hintergrund ahnen kann. Manches erfahren wir aus Stellen, wo er zu sozialen und politischen Umständen seiner Zeit Stellung nimmt.

Augustus wird von Seneca siebenmal in Controversien (2. 4. 12 f.; 2. 5. 20; 4 praef. 5 und 7; 6. 8; 10 praef. 14; 10. 5. 21 f.) und zweimal in Suasorien (1.7; 6. 17) namentlich genannt, meistens in Anekdoten, wo er entweder die pointierte Antwort gibt oder sie selbst bekommt. Ganz oder teilweise gilt diese Charakteristik für sechs aus sieben Erwähnungen in Controversien (2. 5. 20; 4 praef. 5 und 7; 6. 8; 10 praef. 14; 10. $5.21 \mathrm{f}$.). Augustus tritt hier als ein scharfsinniger, beredter und witziger Mann, ${ }^{11}$ der sich für die Redner und ihre Reden persönlich interessiert, ${ }^{12}$ aber auch als ein um seinen Sohn tiefst trauernder und sensitiver ${ }^{13}$ Mann auf. Leicht übertrieben könnte man auch sagen, als „Meister der Bonmots mit einem großen Herzen“. Dies ist ein Augustus-Bild, das wir später z. B. bei Macrobius' Saturnalien beobachten können (Macr. Sat. 2. $4^{14}$ ), das weit von politischen Interessen liegt und sich auf den Privatund Schulbereich konzentriert.

Ein gutes Beispiel für diese Stilisierung von Augustus ist sein Kommentar zur viel zu hohen Geschwindigkeit der Rede von einem gewissen Haterius, und zwar die Empfehlung, einen Hemmschuh einzulegen, um Haterius zu bremsen (Sen. Rhet. Contr. 4 praef. $7^{15}$ ). Der überraschende und ungewöhnliche Kontext des Bremsens deutet auf Augustus' Beredsamkeit hin. Durch das Possesivpronomen noster verleiht Augustus seinem Witz einen väterlichen Ton. Eine ähnliche Kombination von Überraschungsschärfe und familiärer Eleganz der Augustus' Antwort spiegelt sich auch in einer anderen Anekdote wider, in der L. Vinicius als jener gelobt wird, der „Talent in

Eloquence. CSCA 5 [1972] 195-210, hier 196 f., oder DERS.: The Elder Seneca. Lugduni Batavorum 1978, 85-89). Zur Niedergangsdiskussion vgl. auch DöPP, S.: Nec omnia apud priores meliora. Autoren des frühen Prinzipats über die eigene Zeit. RhM 132 (1989) 73-101.

${ }^{11}$ Sen. Rhet. Contr. 2. 5. 20: ... eleganter dixit divus Augustus...; 4 praef. 7: ... divus Augustus optime dixit...

${ }^{12}$ Z.B.: Sen. Rhet. Contr. 2. 4. 12: ... Augusto audiente...; 10 praef. 14: ... Augustus, cum frequenter causas ... audisset...

${ }^{13}$ Sen. Rhet. Contr. 4 praef. 5: ... mortuo in Syria C. Caesare... quaestus esset divus Augustus, ut erat mos illi clementissimo viro, non civiliter tantum sed etiam familiariter, quod in tam magno et recenti luctu suo ... Zu dieser Anekdote siehe weiter.

${ }^{14}$ Macr. Sat. 2. 4. 1: ... , ,Augustus inquam Caesar adfectavit iocos, salvo tamen maiestatis pudorisque respectu, nec ut caderet in scurram. "Nach dieser Einleitung folgen mehr als zwanzig Anekdoten.

${ }^{15}$ Sen. Rhet. Contr. 4 praef. 7: Declamabat autem Haterius admisso populo ex tempore; solus omnium Romanorum, quos modo ipse cognovi, in Latinam linguam transtulit Graecam facultatem. tanta erat illi velocitas orationis, ut vitium fieret. itaque divus Augustus optime dixit: ,Haterius noster sufflaminandus est. ' adeo non currere sed decurrere videbatur. 
bar hat“ (Contr. 2. 5. $\left.20^{16}\right)$, genauso wie in der Augustus’ Anerkennung von Gavius Silo als dem besten „redenden paterfamilias“" (Contr. 10 praef. $14^{17}$ ). Seneca bestätigt in allen erwähnten Fällen, dass die Bemerkungen des Augustus zutrefflich sind, ohne irgendeinen ironischen oder kritischen Unterton auszudrücken oder aus dem Privatbereich ins öffentliche Leben auszutreten. ${ }^{18}$

Aus diesem rein anekdotischen Rahmen in Controversien fallen vier Stellen aus (2. 4.12 f.; 4 praef. 5; mit ein wenig Zweifel 6. 8; und teilweise 10. 5. 21 f.), die zwar auch anekdotische Antworten enthalten, mit weiteren Kommentaren jedoch den Sinn der Aussage über Augustus schon in den öffentlichen Raum rücken. Während in den ersten drei Stellen Augustus als ein nachsichtiger und großzügiger Kaiser gelobt und hochgeschätzt wird, im letzterwähnten Fall, im Buch 10, muss er auch eine unangenehme Rolle übernehmen und einen frechen Rhetor bestrafen. Augustus wird für seine magnitudo und humanitas geschätzt (6. 8), handelt als vir clementissimus (4 praef. 5), admiratione dignus (2. 4. 13), und mit seinen Freunden spricht er nicht nur civiliter, sondern auch familiariter (4 praef. 5) - soweit sieht die Beschreibung also ähnlich wie in den „reinen“ Anekdoten aus. Eben diese Idealisierungen können die immer stärkere Vermutung nicht widerlegen, dass hinter der Fassade etwas anderes steckt.

Im ersten Text, wo Seneca das peinliche Versehen des - seiner Meinung nach ${ }^{19}$ besten Rhetors der augusteischen Zeit und zugleich Senecas Freundes, M. Porcius Latro, beschreibt (2. 4.12 f. $^{20}$ ), werden zwar die Redefreiheit unter Augustus und die

${ }^{16}$ Sen. Rhet. Contr. 2. 5. 20: Hic est L. Vinicius, quo nemo civis Romanus in agendis causis praesentius habuit ingenium: quidquid longa cogitatio alii praestatura erat, prima intentio animi dabat. ex tempore causas agebat, sed non desiderabat hanc commendationem, ut ex tempore agere videretur. de hoc eleganter dixit divus Augustus: ,L. Vinicius ingenium in numerato habet. '

${ }^{17}$ Sen. Rhet. Contr. 10 praef. 14: Bene declamavit Gavius Silo, cui Caesar Augustus, cum frequenter causas agentem in Tarraconensi colonia audisset, plenum testimonium reddidit; dixit enim: numquam audivi patrem familiae disertiorem.

${ }^{18}$ Für irreführend halte ich die politische Interpretation der zweiten der angeführten Anekdoten (Contr. 2. 5. 20), die in einer sonst sehr umfangreichen und tiefen Studie von M. TORRI (La réception de la propagande d'Auguste chez Sénèque le Rhéteur. Classica 15/16 [2002/2003] 117-130, ,une certain ambiguité et de l'ironie": S. 120) enthalten ist. Diese Interpretation beruht auf dem Argument, dass die für Rom einmalige Verbindung von Eleganz und Geld eine kritische Andeutung auf die Kleinlichkeit oder etwa Sparsamkeit von Augustus darstellt (,le prince est montré avec son penchant pour l'argent“: S. 120). Der eben angedeutete Zusammenhang dieser Anekdote mit den beiden weiteren, die auf einem vergleichbaren Spiel mit einer unerwarteten Metaphorik und außergewöhnlichen Ausdrücken (in der Datenbank BTL erscheint der Ausdruck in numerato [2. 5. 20] ebenso selten wie sufflaminandus [10 praef. 14]) begründet sind, widerlegt eine politische Auslegung. Eine unerwünschte Beziehung des Augustus zu Geld wird von den Historikern keineswegs signalisiert, im Gegenteil: Sueton bezeichnet Augustus wiederholt als sehr freigebig (vgl. Suet. Aug. 30 oder 41).

${ }^{19}$ Siehe Seneca's Contr. 10 praef. 13: Primum tetradeum quod faciam, quaeritis! Latronis, Fusci [cesti], Albuci, Gallionis.

${ }^{20}$ Sen. Rhet. Contr. 2. 4. 12 f.: In hac controversia Latro contrariam rem 〈non〉 controversiae dixit sed sibi. declamabat illam Caesare Augusto audiente et M. Agrippa, cuius filios, nepotes suos, Caesar [Lucium et Gaium] adoptaturus diebus illis videbatur. erat M. Agrippa inter eos, qui non nati sunt nobiles sed facti. cum diceret partem adulescentis Latro et tractaret adoptionis locum, dixit: non asciti ex imo per adoptionem nobilitati 〈in〉serunt〈ur, et〉 [in hanc] alia in hanc summam. Maecenas innuit Latro〈ni〉 festinare Caesarem; finiret iam declamationem. quidam putabant hanc malignam rem Maecenatis esse; effecisse enim illum, non ne audiret quae dicta erant Caesar, sed ut notaret. / Tanta autem [marcus] sub 
Großzügigkeit des Kaisers hervorgehoben und es werden auch zwei konkrete Beispiele unbestrafter Impertinenz angegeben, aus Senecas letzten Worten dieses Absatzes wird jedoch klar, dass die Grenze dieser Freiheit sehr zerbrechlich ist. Aufs Spiel wird dabei nichts Kleineres als der Kopf gesetzt.

Seneca beginnt die ganze Passage mit der Beschreibung des Kontextes der Rede, die den Latro in eine sehr unangenehme Situation bringt. Bei der Vorführung der Argumente zugunsten des Jünglings, der seinen Vater anklagt, den Sohn aus dem Bund seines früher enterbten Bruders mit einer Prostituierten als seinen Enkel anerkannt zu haben, hat Latro die Tatsache übersehen, dass vor ihm nicht nur Augustus sitzt, von dessen edler Herkunft kein Zweifel bestehen konnte, sondern auch Augustus' unadeliger Schwiegersohn M. Vipsanius Agrippa, dessen Söhne Augustus im Begriff war zu adoptieren, und somit ihre mögliche Nachfolgerschaft zu sichern. Latro führt an, dass die Leute ,aus der Unterschicht“ (ex imo) nicht aufgrund einer Adoption zur Nobilität erhoben werden sollten. Seneca entschuldigt den Latro und als den Anstifter der ganzen Unannehmlichkeit bezeichnet er den Maecenas, der nach Seneca unmittelbar nach diesen Worten den Latro absichtlich unterbrach, nicht um ihn vor einem möglichen Skandal zu schützen, sondern im Gegenteil, um den möglichen Bezug seiner Rede zu Augustus und Agrippa hervortreten zu lassen, der unter anderem eine schwerwiegende Beleidigung von Augustus' Tochter (durch den Vergleich mit einer Prostituierten) bedeuten würde. ${ }^{21}$ Gegenüber dem bedauernswerten Latro, der sich für die unbeabsichtigte Beleidigung nicht entschuldigen konnte, stellt folgend Seneca ungenannte Wagehälse, die mit den Späßen auf Kosten von Agrippa in Verteidigerrolle ihrer Klagerede an Kraft zusetzen wollten. Seneca stellt fest, dass er kein Erbarmen mit jenen haben kann, die wegen sprachlicher Äquilibristik ihr Leben aufs Spiel setzen (qui tanti putant caput potius quam dictum perdere). Augustus wird hier zwar als eine bewundernswerte liberale Persönlichkeit hervorgehoben und das Maß an Freiheit wird gleich zweimal als hoch bewertet (tanta... libertas; und später admiratione dignus divus Augustus, sub quo tantum licuit), dennoch wird allein aus dem Schluss ersichtlich, dass man bereits unter Augustus für schärfere Worte, die im Rahmen eines Gerichtsverfahrens früher ganz geläufig waren, „,den Kopf verlieren“ konnte. ${ }^{22}$

divo Augusto libertas fuit, ut praepotenti tunc M. Agrippae non defuerint qui ignobilitatem exprobrarent. Vipsanius Agrippa fuerat, 〈at〉 Vipsani nomen quasi argumentum paternae humilitatis sustulerat et $M$. Agrippa dicebatur. cum defenderet reum, fuit accusator qui diceret: , Agrippae, Marce et quod in medio est' (voluit Vipsanium intellegi), [fuit qui diceret] ,concurrite, Agrippae: malum habebi〈ti〉s, ‘nisi〉 responderitis $\langle a d\rangle$ ea, Marce, alterque!' mihi videtur admiratione dignus divus Augustus, sub quo tantum licuit, sed horum non possum misereri, qui tanti putant caput potius quam dictum perdere. Latro dignus fuit miseratione, qui ne excusare quidem errorem suum potuit. nihil est autem crudelius quam sic offendere, ut magis sis offensurus, si satis feceris.

${ }^{21}$ Wenn überhaupt über einen Adressaten dieser unschicklichen Beleidigung die Rede sein kann, dann ist er meiner Meinung nach direkt Augustus und seine Tochter, die Mutter beider Knaben von Agrippa, und nicht Agrippa und seine Mutter (wie es von TORRI [Anm. 18] 119 f. wahrgenommen wird), wiewohl die weiteren Worte Senecas lediglich zu den Beleidigungen von Agrippa bezogen werden.

${ }^{22}$ Vgl. jedoch TORRI (Anm. 18) 220, der den Kommentar Senecas umgekehrt als Beweis wahrnimmt, dass zwischen den Jahren, in welche das Ereignis gesetzt werden kann, und dem Jahr $40 \mathrm{n}$. Chr. Augustus nicht unantastbar war. In ganz anderen Zusammenhängen - als eine literarische Allegorie, welche die Verbindung des realen Lebens mit der Dekadenz abbildet - bewertet dieses Ereignis und auch 
In den politischen Zusammenhang werden die Gerichtsreden und sogar die bislang ganz harmlosen Übungsreden gebracht. ${ }^{23}$

Im zweiten Text erwähnt Seneca im Rahmen des Porträts von C. Asinius Pollio seinen kleinen Wortaustausch mit Augustus (Contr. 4 praef. $5^{24}$ ). Als Augustus erfährt, dass Pollio nach dem Tod des vom Prinzeps adoptierten Sohnes Gaius nicht die Trauer gehalten und am Gelage teilgenommen hat, schreibt er an Pollio ein Schreiben, wo er ihn darüber eher väterlich und freundlich schilt. Als sein wertester (carissimus) Freund hätte Pollio den Augustus in seiner so großen und frischen Trauer (in tam magno et recenti luctu) stützen sollen. Pollios lakonische Antwort, dass er auch an dem Tag geschmaust hat, als er seinen eigenen Sohn verloren hatte, erteilte dem Augustus eine Art von moralischer Ohrfeige, die Seneca durch die rhetorische Frage potenziert, wer vom Freund größeren Schmerz verlangen würde als vom Vater. Anhand dieser Geschichte bezeugt Seneca die bewundernswerte Kraft und Festigkeit der Persönlichkeit von Pollio, die es ihm möglich machte, seinen Kummer zu leugnen und kurz nach dem Tod seines Sohnes sogar öffentlich zu reden, laut Seneca im hartnäckigen Trotz gegen das Schicksal (4 praef. 4: ... tanto vehementius quam umquam, ut appareret hominem natura contumacem cum fortuna sua rixari). Obwohl Seneca als Gegenpol zu Pollio den pathetisch, ungemäß lange trauernden Redner Q. Haterius stellt (4 praef. 6), kann man sich nicht des Eindrucks erwehren, dass er heimlich auch auf Augustus zielt, der in seiner Empörung und Schwäche eine typisch autokratische Eigenschaft bloßstellte, wenn er erwartete, dass seine persönliche Trauer als bürgerliche Trauer aufgenommen werden wird (die Verbindung mit dem Bürgerleben spiegelt

manche weiteren Erwähnungen über Augustus GUNDERSON, E.: Declamation, Paternity, and Roman Identity: Authority and the Rhetorical Self. Cambridge 2003, insb. Kap. 3, S. 90-115. In diesem konkreten Fall kann nach Gunderson das ganze Ereignis übertragen als eine Aufforderung für Augustus zur Wahl seiner Rolle in Richtung Rolle des literarischen Kritikers aufgefasst werden (und die folgende Schätzung des Augustus von Seneca, dass er gerade eine solche Rolle - die Nichtbestrafung Latros - akzeptierte; vgl. ibid. 101-104). Die weitere Entfaltung dieser Überlegung, dass nämlich Seneca durch die Schilderungen des Augustus als des Zuhörers von Deklamationen eine Situation schafft, in der sich seine Autorität als Erzähler an der tatsächlichen Autorität des Kaisers beteiligt (ibid. 103), geht aus meiner Sicht schon zu weit und entspricht nicht mehr der Realität.

${ }^{23}$ Vgl. in diesem Zusammenhang die höchst interessante Studie über das Verhältnis des Kaisers und der Deklamationen von K. VössING (Der Kaiser und die Deklamationen. In PERRIN, Y. [Hrsg.]: $N e-$ ronia VIII. Bibliothèques, livres et culture écrite dans l'empire romain de César à Hadrien; Actes du VIII Coll. internat. de la SIEN (Paris 2008). Bruxelles 2010, 301-314), die von dieser Episode ausgeht und sich mehr allgemein den Änderungen in der Auffassung der Deklamationen in der augusteischen Zeit widmet. Eine solche Änderung besteht eben in der Übertragung ,nicht-öffentlicher otium-Beschäftigung der Erwachsenen... zu einer Form öffentlicher Präsentation“ (S. 305). Aus ähnlicher Sicht analysiert die Reden im Prinzipat M. Lentano (Die Stadt der Gerichte. Das Öffentliche und das Private in der römischen Deklamation. In Haltenhoff, A. - Heil, A. - Mutschler, FR.-H.: Römische Werte und römische Literatur im frühen Prinzipat. Berlin 2011, 209-232). Im Kontext der hier analysierten Stelle soll seine Beobachtung erwähnt werden, dass die Anwesenheit Augustus' als Beweis für Augustus' Willen gilt, die rednerischen Schulen unter Kontrolle zu halten (ibid. 222).

${ }^{24}$ Sen. Rhet. Contr. 4 praef. 5: Itaque cum mortuo in Syria C. Caesare per codicillos quaestus esset divus Augustus, ut erat mos illi clementissimo viro, non civiliter tantum sed etiam familiariter, quod in tam magno et recenti luctu suo homo carissimus sibi pleno convivio cenasset, rescripsit Pollio: ,eo die cenavi, quo Herium filium amisi. ' quis exigeret maiorem ab amico dolorem quam a patre? 
sich eben in der bereits zitierten Verbindung non civiliter tantum, sed etiam familiariter wider). ${ }^{25}$

Ebenso die dritte Passage beschreibt (Contr. 6. $8^{26}$ ) - zwischen den Zeilen die Unsicherheit der Redner, die zögern, vor Augustus offiziell aufzutreten, weil sie nicht wissen, was sie vom princeps erwarten können. In dieser Passage wird eine kluge Sentenz von Varius Geminus wiederholt (,diejenigen, die es wagen, vor dir, Kaiser, zu reden, kennen deine Größe nicht, die es aber nicht wagen, kennen deine Vornehmheit nicht"). ${ }^{27}$

Schließlich, in der vierten Passage (Contr. 10. 5. $21 \mathrm{f}^{28}$ ), erfahren wir von einer für das Prinzipat immer mehr typischen Blitzkarriere, und zwar eines Kriegsgefangenen Timagenes, der Koch, Sänftenträger und letztendlich auch Freund von Augustus wurde, der allerdings, wie Seneca klar andeutet, mit seiner schnell erworbenen Position infolge seiner zu lang andauernden Sklavenschaft nicht umgehen konnte (nimis liber erat - puto, quia diu non fuerat) und zum Schluss aus dem Augustus' Palast verbannt wurde. ${ }^{29}$ Auch wenn Seneca nur wenig Verständnis für Timagenes hat (ausgenommen seine rednerische Qualität: a quo multa improbe sed venuste dicta) und ihn als undankbaren, sauren und frechen Mann beschreibt (homo acidae linguae; dicax), macht er zugleich klar, dass für eine Verbannung hinreichend war, wenn Augustus seine Gründe hatte, iratus zu sein. Eine gewisse Bissigkeit kann man überdies auch in der Beschreibung des raschen gesellschaftlichen Aufstiegs nicht übersehen, in deren Subtext sich die Empörung darüber widerspiegelt, auf welche Weise sich Augustus seinen Fördererkreis sicherte und mit welcher Gesellschaft er sich umgab.

In den zwei bereits erwähnten Stellen in Suasorien, in denen der Name Caesar direkt auftritt, kommt die Person von Augustus nur nebenbei vor - in Kommentaren von dritten Personen (Messala und Livius), und zwar schon ganz außerhalb des anekdotischen Rahmens, aber - ähnlich wie bei den soeben analysierten vier Erwähnun-

${ }^{25} \mathrm{Vgl}$. auch hier eine unterschiedliche, literarisch analytische Sicht auf diese Begebenheit bei GUNDERSON (Anm. 22) 96-99. Wiewohl Gundersons Analysen eine interessante Erfrischung der klassischen Interpretation bringen, zuweilen ,versenken“ sie in der Tat den ursprünglichen Text in den Tiefen der Psychoanalyse und verzerren die Gattung der Deklamationen.

${ }^{26}$ Sen. Rhet. Contr. 6. 8: Varius Geminus apud Caesarem dixit: Caesar, qui apud te audent dicere, magnitudinem tuam ignorant, qui non audent, humanitatem.

${ }^{27}$ In diesem Zusammenhang muss unbedingt eine ähnliche, jedoch mehr explizite Äußerung, erhalten bei Macrobius, erwähnt werden: Macr. Sat. 2. 4. 21: Temporibus triumviralibus Pollio, cum Fescenninos in eum Augustus scripsisset, ait: at ego taceo. non est enim facile in eum scribere qui potest proscribere. Vgl. abermals auch eine andere Auffassung der Geminus-Sentenz: GUNDERSON (Anm. 22) 103.

${ }^{28}$ Sen. Rhet. Contr. 10. 5. 21 f.: ... Hic est Craton, venustissimus homo et [pro homo et] et professus Asianus, qui bellum cum omnibus Atticis gerebat. Cum donaret illi Caesar talentum, in quo viginti

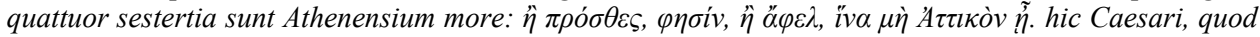
illum numquam nisi mense Decembri audiret, dixit: $\omega \varsigma \beta \alpha v ́ \omega \mu$ ol $\chi \rho \tilde{\eta}$; et 〈cum〉 commendaretur a Cae-

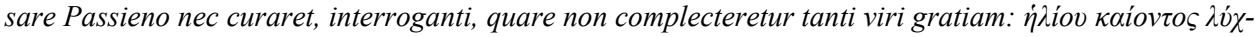

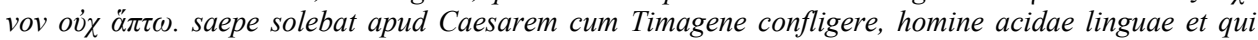
nimis liber erat - puto, quia diu non fuerat. Ex captivo cocus, ex coco lecticarius, ex lecticario usque in amicitiam Caesaris †felix† usque eo utram‘que fortunam contempsit, et in qua erat et in qua fuerat, ut, cum illi multis de causis iratus Caesar interdixisset domo, combureret historias rerum ab illo gestarum, quasi et ipse illi ingenio interdiceret; disertus homo et dicax, a quo multa improbe sed venuste dicta.

${ }^{29} \mathrm{Vgl}$. des Interesses halber wiedermal auch GuNDERSON (Anm. 22) $94 \mathrm{ff}$. 
gen - ist hier der kritische Subtext sichtbar. Die Fahnenflucht von Dellius, der nicht zögerte, gleich dreimal das politische Lager zu wechseln, bis er endlich unter den Anhängern von Augustus landete (Suas. 1. $7^{30}$ ), ist nicht nur ein Zeichen für die unsichere Zeit und dessen unfesten Charakter, sondern, ähnlich wie bei der letztbehandelten Erwähnung von Controversien, ist es auch ein gewisser Stachel gegen Augustus und seinen Geschmack, oder zumindest wohl gegen die allzu niedrigen Ansprüche an die Anhänger, und dies einschließlich seiner Kollegen Triumvirn. Die Zeit der Triumviratssäuberungen wird uns auch durch die von Cicero wiedergegebene Befürchtung nähergebracht, dass ihm nicht gelingt, dem Antonius zu entgehen, so wie Brutus und Cassius dem Augustus nicht entgangen sind (Suas. 6. 17 $7^{31}$ ). Wiewohl dieser verhängnisvolle Augenblick im Leser einen Unwillen gegenüber Antonius, und nicht gegenüber Augustus erweckt, überträgt der angegebene Vergleich einen gewissen Anteil von Verantwortung für Ciceros Tod (und nicht nur dafür) eindeutig auch auf ihn. ${ }^{32}$ Zur Ciceros Rolle in Senecas Kritik an Augustus komme ich später noch zurück.

Während in den Beschreibungen der Ereignisse, die Augustus direkt betreffen, wie gerade gezeigt wurde, Seneca diplomatisch vorgeht und die ohnehin milde Äußerungen noch mehr schwächt, (z.B. wenn er die Betroffenen als diejenigen beschreibt, die die Strafe sowieso verdient haben: homo acidae linguae, dicax, improbe dicens, d.h. die Verantwortung auf die Opfer zu übertragen versucht), die indirekten Bemerkungen zeigen etwas mehr Mut und verraten sogar Zorn. Auch wenn hier Augustus nie offen angesprochen wird, ist es klar, dass sich vieles vom Gesagten und Gefühlten auf ihn bezieht.

In diesem Zusammenhang ist ein Teil der Vorrede zum letzten Buch der Controversiae wohl am bekanntesten $\left(10\right.$ praef. $\left.5-8^{33}\right)$. Seneca wird von seinen Söhnen nach

${ }^{30}$ Sen. Rhet. Suas. 1. 7: ... Dellius... quem Messala Corvinus desultorem bellorum civilium vocat, quia ab Dolabella ad Cassium transiturus salutem sibi pactus est, si Dolabellam occidisset, a Cassio deinde transit ad Antonium, novissime ab Antonio transfugit ad Caesarem.

${ }^{31}$ Sen. Rhet. Suas. 6. 17: M. Cicero... pro certo habens, id quod erat, non magis Antonio 〈se〉 eripi quam Caesari Cassium et Brutum posse.

${ }^{32}$ In Bezug auf die absichtliche Verbindung zwischen Augustus und Antonius sowie auf die Expressivität des Verbes se eripere scheint die Meinung von P. WITZMANN (Num recte sit Livius ab imperatore Augusto Pompeianus appellatus. Ein Fall möglicher Ambivalenz im Liviustext. In HALTENHOFFHEIL-MuTSCHLER [Anm. 23] 81-110, hier 104), dass Seneca sehr beachtet, auf Augustus keinen Schatten fallen zu lassen, unangemessen.

${ }^{33}$ Sen. Rhet. Contr. 10 praef. 5-8: In hoc [T. Labieno: KP] primum excogitata est nova poena: effectum est enim per inimicos, ut omnes eius libri comburerentur. res nova et inusitata, supplicium de studiis sumi. bono hercules publico ista in poenas ingeni versa crudelitas post Ciceronem inventa est. quid enim futurum fuit, si triumviris libuisset et ingenium Ciceronis proscribere? sunt di immortales lenti quidem sed certi vindices generis humani et mala exempla in caput invenientium regerunt, ac iustissima patiendi vice quod quisque alieno excogitavit supplicio saepe imitat〈ur suo. quae vos, dementissimi homines, tanta vecordia agitat? parum videlicet in poenas notae crudelitatis est: conquirite in vosmet ipsos nova, quibus pereatis, et si quid ab omni patientia rerum natura subduxit, sicut ingenium memoriamque nominis, invenite, quemadmodum reducatis ad eadem rem corporis mala[...].[...] eius, qui hanc in scripta Labieni sententiam dixerat, postea viventis adhuc scripta combusta sunt, iam non malo exemplo, quia suo. Non tulit hanc Labienus contumeliam nec superstes esse ingenio suo voluit sed in monumenta se maiorum suorum ferri iussit atque ita includi, veritus scilicet, ne ignis, qui nomini suo subiectus erat, corpori negaretur. non finivit tantum se ipse sed etiam sepelivit. Memini aliquando, cum recitaret historiam, 
T. Labienus gefragt. Nach einer ziemlich kritischen Beschreibung von Labienus' Härte, Knallkraft und Freiheit, die alle zugelassenen Grenzen übersteigen (Contr. 10 praef. 4), wobei Seneca dem Labienus, mit dem Beinamen Rabienus, immerhin ein großes Talent zuschreibt, kommt eine besonders scharfe Verurteilung der Verbrennung von Labienus' Büchern. ${ }^{34}$ Seneca benutzt besonders starke Wörter: diejenigen, die über die Strafe entschieden haben, d.h. die Senatoren, betitelt er mit dementissimi homines, das Urteil selbst vecordia. (Hier will ich darauf hinweisen, dass Cicero so ein starkes Wort nur einmal benutzt hat und das Wort auch allgemein mindestens zur Senecas Zeit sehr rar war. ${ }^{35}$ ) Seneca fügt sogar eine Bedrohung hinzu, so ein Verhalten bleibe nicht unbestraft von Göttern (sunt di immortales lenti quidem sed certi vindices generis humani et mala exempla in caput invenientium regerunt...). Am Ende verwandelt sich die höchste Empörung durch eine Anekdote des Severus Cassius in die Ironie (Contr. 10 praef. $8^{36}$ ), die die Senatorenentscheidung herabsetzt und somit insgesamt den Zustand des öffentlichen Lebens unter Augustus kritisiert.

Interessant ist schon die diesem scharf kritischen Kommentar vorangehende Einleitung, wo sich eine düstere Atmosphäre spürbar macht. Seneca empfindet Überdruss, fühlt sich belastet, schreibt fast nur aus Zwang, nur um die Aufgabe definitiv loszuwerden (Contr. 10 praef. $1^{37}$ ). Meiner Meinung nach wählt Seneca solche Einleitung absichtlich, um als ein alter, müder und harmloser Mann frei reden zu können und seine Worte auf emotionaler Ebene zu unterstreichen. Auch die Reaktion des Severus Cassius ist hier nicht umsonst erwähnt: Sein Schicksal war sehr ähnlich wie das des Labienus, wobei diesmal der Senat nicht nur seine Bücher verbrannt, sondern auch den Autor mit Exil bestraft hat. ${ }^{38}$ Diese Tatsache lässt Seneca in seinen Ausführungen aus. Er hat es aber gar nicht sagen müssen. Ich bin mir sicher, dass dies dem Leser sofort aufgefallen ist. Schweigen ist hier stärker als Reden. Und noch etwas. Seneca zeigt, dass die Flammen nicht vermögen, die Öffentlichkeit zum Schweigen zu bringen. Im Gegenteil. Die beiden Autoren werden von ihm - sporadisch, aber immerhin zitiert $^{39}$ und bei Seneca ist sogar eine längere Rede des Cassius Severus erhalten

magnam partem illum libri convolvisse et dixisse: haec quae transeo, post mortem meam legentur. quanta in illis libertas fuit, quam etiam Labienus extimuit!

${ }^{34}$ Breitere Zusammenhänge zu dieser Begebenheit, d.h. zur Strafe mit Bücherverbrennung, siehe CRAMER, FR. H.: Bookburning and Censorship in Ancient Rome: A Chapter from the History of Freedom of Speech. Journal of the History of Ideas 6.2 (1945) 157-196, insb. 172-175; und neulich insb. BORGO, A.: Res nova et inusitata, supplicium de studiis sumi (Sen. Contr. 10 praef. 5). A proposito dei roghi di libri a Roma. Paideia 67 (2012) 33-53.

${ }^{35}$ In BTL gibt es nur 26 Belege vor Seneca aus insgesamt 109 Erscheinungen.

${ }^{36}$ Sen. Rhet. Contr. 10 praef. 8: ... Cassi Severi, hominis Labieno invissimi, belle dicta res ferebatur illo tempore, quo libri Labieni ex senatus consulto urebantur: nunc me, inquit, vivum uri oportet, qui illos edidici.

${ }^{37} \mathrm{Vgl}$. Anm. 9 in diesem Beitrag.

${ }^{38}$ Siehe CRAMER (Anm. 34) 175 ff. Vgl. auch Tac. Ann. 1. 72. 3: Tacitus stellt hier Augustus' Entscheidung, auch Worte nach lex maiestatis zu verfolgen, in Zusammenhang eben mit Cassio.

${ }^{39}$ T. Labienus, Contr. 4 praef. $2 ; 10$ praef. $8 ; 10.2 .19 ; 10.3 .5$ und 15; 10. 4.17 f. und 24 f. Cassius Severus, Contr. 2. 4. 11; 3 praef.; 7. 3.8 und 10; 9. 2. 12; 9. 3. 14; 10 praef. 8; 10. 4. 2 und 25; 10. 5. 20; Suas. 6. 11. 
(Contr. 3 praef. 8-17). In dieser wird argumentiert, warum Deklamationen und Deklamatoren nie so gut sein können wie Forumreden und -redner ${ }^{40}$ - dasselbe vertritt auch Seneca in den einleitenden Worten $^{41} \mathrm{zu}$ seiner schärfsten gegenaugusteischen Passage, die eng mit der Trauer über den Verlust der Demokratie zusammenhängt.

Vieles über Senecas Gefühle, was die Zeit des kommenden Prinzipats betrifft, erfahren wir aus der Vorrede zum zweiten Buch (Contr. 2 praef. 3 f. ${ }^{42}$ ). Seneca wendet sich an seinen jüngsten Sohn und lobt ihn, dass er keine öffentliche Karriere machen will. Er ruft Mela zwar auf, rednerische Kunst weiter zu pflegen, aber nur aus dem Grunde, um weitere Künste leichter beherrschen zu können und zum kultivierten Mann zu werden (facilis ab hac [i.e. eloquentia] in omnes artes discursus est). Seneca macht sich riesige Sorgen um seine anderen Söhne, die am öffentlichen Leben teilnehmen wollen und sich auf forum und honores, in quibus ipsa quae sperantur timenda sunt vorbereiten, also ist er glücklich, dass mindestens sein jüngster Sohn im Sicheren, in portu, bleibt.

Auch wenn sich Senecas Ansprache höchstwahrscheinlich erst auf die Zeit nach Augustus bezieht, unter Augustus ging es wohl nicht anders zu. Eine gewisse Lebenssicherheit konnte dem Redner nur solche Rhetorik bieten, die als scholastische oder noch besser propädeutische Disziplin die Schüler auf weitere Studien vorbereitet hat. Diese konnte jedoch der ,wahren“ Rhetorik nicht konkurrieren, wie Cassius Severus (siehe oben) oder Votienus Montanus (Contr. 9 praef. $1-5^{43}$ ) bei Seneca sehr lebhaft

${ }^{40}$ Vgl. vor allem Sen. Rhet. Contr. 3 praef. $12 \mathrm{ff} .:$,, ... cum in foro dico, aliquid ago; cum declamo, id quod bellissime Censorinus aiebat de his, qui honores in municipiis ambitiose peterent, videor mihi in somnis laborare. deinde res ipsa diversa est: totum aliud est pugnare, aliud ventilare. hoc ita semper habitum est, scholam quasi ludum esse, forum arenam, et ille ideo primum in foro verba fracturus tiro dictus est. agedum istos declamatores produc in senatum, in forum: cum loco mutabunt «ur. velut adsueta clauso et delicatae umbrae corpora sub divo stare non possunt, non imbrem ferre, non solem sciunt; vix se inveniunt. adsuerunt enim suo arbitrio diserti esse. non est, quod oratorem in hac puerili exercitatione spectes. quid, si velis gubernatorem in piscina aestimare?..."

${ }^{41}$ Sen. Rhet. Contr. 10 praef. 1: ... deinde me iam pudet, tamquam diu non seriam rem agam. hoc habent scholasticorum studia: leviter tacta delectant, contrectata et propius admota fastidio sunt.

${ }^{42}$ Sen. Contr. 2 praef. 3 f.: ... Haec eo libentius, Mela, fili carissime, refero, quia video animum tuum a civilibus officiis abhorrentem et ab omni ambitu aversum hoc unum concupiscentem, nihil concupiscere - ut eloquentiae tamen studeas. facilis ab hac in omnes artes discursus est; instruit etiam quos non sibi exercet. nec est, quod insidias tibi putes fieri, quasi id agam, ut te bene cedentis studii favor teneat; ego vero non sum bonae mentis impedimentum: perge quo inclinat animus, et paterno contentus ordine subduc fortunae magnam tui partem. erat quidem tibi maius ingenium quam fratribus tuis, omnium bonarum artium capacissimum; est et hoc ipsum melioris ingenii pignus non corrumpi bonitate eius, ut illo male utaris. sed quoniam fratribus tuis ambitiosa curae sunt foroque se et honoribus parant, in quibus ipsa quae sperantur timenda sunt, ego quoque, eius alioqui processus avidus et hortator laudatorque vel periculosae dum honestae modo industriae, duobus filiis navigantibus te in portu retineo.

${ }^{43}$ Sen. Rhet. Contr. 9 praef. 1 f.: MONTANVS VOTIENVS adeo numquam ostentationis ‘de〉clamavit causa, ut ne exercitation is quidem declamaverit. rationem quaerenti mihi ait: utram vis, honestam an veram? si honestam, ne 〈***; si veram, ne〉 male adsuescam. qui declamationem parat, scribit non ut vincat sed ut placeat. omnia itaque lenocinia [ita] conquirit; argumentationes, quia molestae sunt et minimum habent floris, relinquit. sententiis, explicationibus audientis delinire contentus est. cupit enim se approbare, non causam. sequitur autem hoc usque in forum declamatores vitium, ut necessaria deserant, dum speciosa sectantur. Die Auslegung setzt mit der Beschreibung weiterer Mängel der Deklama- 
beschrieben haben. Ich glaube, gerade der sich immer vergrößernde Abstand zwischen Rede und Deklamation und der Realitätsverlust der Deklamation stellen für Seneca das Symbol der unsicheren und unvorhersehbaren Zeit dar, die, auch wenn es Seneca nie direkt ausspricht oder nur andeutet, von seiner idealen Vorstellung weit entfernt ist.

Als letzten Beweis für diese Behauptung nehme ich Cicero und seine Rolle in Senecas Beziehung zur Augustus' Politik. Schon in der Vorrede verkörpert Cicero für Seneca den Gipfel der rhetorischen Kunst, nach welchem es zum kontinuierlichen Verfall kam (Contr. 1 praef. $6 \mathrm{f}^{44}$ ). Zugleich bedeutet aber Cicero den Anfang der rhetorischen Übungen, die Deklamationen benannt wurden (Contr. 1 praef. $12^{45}$ ). Später erspart sich Seneca nicht die bissige Bemerkung, dass Calvus lange Zeit vergeblich versucht hat, Ciceros rednerischen Qualitäten nachzukommen (Contr. 7. 4. $6^{46}$ ). Seneca hält sich aber nicht ausschließlich in den Grenzen der Rhetorik. In Controversien ist ein Kapitel dem moralischen Hintergrund des Todes von Cicero durch die Hand seines Klienten Popillius gewidmet (Contr. 7. $2^{47}$ ). In Suasorien stellt sich sogar Cicero als politische Persönlichkeit zwei Fragen, und beide beziehen sich auf Antonius. Er überlegt, ob er Antonius folgen und sich entschuldigen sollte bzw. ob er seine Bücher vernichten sollte, um von Antonius Gnade zu erhalten (Suas. 2. 6- $7^{48}$ ). Im Zusammenhang mit der Verbrennung von Labienus' Büchern bemerkt Seneca sarkastisch, dass ein riesiges Glück war, dass diese Strafe erst nach Cicero eingeführt wurde, denn sonst wäre auch sein ingenium von den Triumvirn nach Beliebigkeit proskribiert worden (Contr. 10 praef. 6: bono hercules publico ista in poenas ingeni versa crudelitas post Ciceronem inventa est. quid enim futurum fuit, si triumviris libuisset et ingenium Ciceronis proscribere ${ }^{49}$ ). Auch wenn es Seneca nur mit zwei kurzen Sätzen tut, beschreibt er hier doch sehr naturalistisch die Willkür des Triumvirats, an dem Augustus teilnahm.

Ganz zuletzt möchte ich einen höchst bemerkenswerten Absatz in der Vorrede besprechen, der Cicero gewidmet ist (Contr. 1 praef. $11^{50}$ ). Ich will aber nicht auf das

tionen fort, einschließlich eines anschaulichen Belegs von der negativen Wirkung der Deklamation, nämlich der Unfähigkeit des sonst hervorragenden Deklamators M. Porcius Latro, in einer realen Causa auf dem Forum zu reden.

${ }^{44}$ Sen. Rhet. Contr. 1 praef. 6 f.: ... quidquid Romana facundia habet, quod insolenti Graeciae aut opponat aut praeferat, circa Ciceronem effloruit; omnia ingenia, quae lucem studiis nostris attulerunt, tunc nata sunt.

${ }^{45}$ In Contr. 1 praef. 12 vgl. vor allem: ... ipsa ,declamatio ' apud nullum antiquum auctorem ante Ciceronem et Calvum inveniri potest...

${ }^{46}$ Sen. Rhet. Contr. 7. 4. 6: ... CALVVS, qui diu cum Cicerone iniquissimam litem de principatu eloquentiae habuit...

${ }^{47}$ Sen. Rhet. Contr. 7. 2: DE MORIBVS SIT ACTIO. Popillium parricidii reum Cicero defendit; absolutus est. proscriptum Ciceronem ab Antonio missus occidit Popillius et caput eis ad Antonium rettulit. Accusatur de moribus.

${ }^{48}$ Sen. Suas. 2. 6: Deliberat Cicero, an Antonium deprecetur. 2. 7: Deliberat Cicero, an scripta sua comburat promittente Antonio incolumitatem, si fecisset.

${ }^{49}$ Siehe oben Anm. 33.

${ }^{50}$ Sen. Rhet. Contr. 1 praef. 11: ... alioqui in illo atriolo, in quo duos grandes praetextatos ait [i.e. Cicero] secum declamare, potui adesse illudque ingenium, quod solum populus Romanus par impe- 
schön aufgebaute Lob des Redners näher eingehen, sondern auf eine unauffällige Notiz. In dieser wird gesagt, dass Cicero in einem kleinen Atrium mit zwei großen Senatoren deklamiert hat. Die Senatoren waren, wie es schon erwähnt wurde, ${ }^{51}$ zwei für das Jahr 43 designierte Konsuln Hirtius und Pansa. Beide waren Anhänger von Caesar und nach seinem Tod hätten eher zu Antonius inkliniert. Und hier hat Cicero vermutlich eine wichtige Rolle gespielt. Er soll beide - sicherlich Hirtius, vielleicht auch Pansa - überredet haben, zu den Senatoren überzugehen; beide sind dann im Kampf ums Leben gekommen (nach Sueton sogar vielleicht mit direktem Anteil des Augustus: vgl. Suet. Caes. Aug. 11; Tac. Ann. 1. 10. 1-2). Cicero hat darüber mehrere Briefe geschrieben, in denen er verschiedene Schlingen der diplomatischen Verhandlung dokumentiert hat. ${ }^{52}$ Will Seneca mit diesem - ansonsten völlig unnötigen - Detail den Leser eben auf diesen letzten Versuch aufmerksam machen, die römische Demokratie zu erhalten? Will etwa Seneca zugleich seine Trauer darüber zum Ausdruck bringen, dass dieser Versuch vergeblich war und dass Cicero von Augustus geopfert wurde, auch wenn er mehr oder weniger zu seinem Gunsten ${ }^{53}$ gehandelt hat? Ich lasse die Fragen offen, glaube jedoch, die Antwort bereits angedeutet zu haben.

Meiner Meinung nach kann angenommen werden, dass Seneca den Neuheiten unter Augustus nicht zugestimmt hat, dass er jedoch, wie die Meisten, nicht gewagt hat, sich gegen Augustus - sei es nach seinem Tod - öffentlich zu stellen. Er war vernünftig genug, um zu verstehen, dass unter Umständen das Leben und die pax Romana mehr als die Freiheit bedeuten. ${ }^{54}$ Er hat jedoch den Weg gefunden, seine Sympathien bzw. Antipathien auszusprechen und seinen Söhnen indirekt ein politisches „Testament“ zu hinterlassen. ${ }^{55}$ Es wäre höchst interessant, über sein historisches Werk

rio suo habuit, cognoscere et, quod vulgo aliquando dici solet sed in illo proprie debet, potui vivam vocem audire. Für die ganze Passage siehe Anfang dieses Absatzes.

${ }_{52}^{51}$ Siehe oben Anm. 5.

${ }^{52}$ Siehe eine detaillierte Online-Studie von CHAMBERS, E.: Having Hirtius to Dinner: Optimates and Populares in the Late Republic. Eras Journal 3 (2002). Available from URL: http://www.arts.monash. edu.au/publications/eras/edition-3/chambers.php.

${ }^{53}$ Die Beziehung zwischen Cicero und Augustus war gewiss nicht unproblematisch. Cicero hat den Augustus zwar unterstützt, aber gleichzeitig war er sich dessen bewusst, dass Augustus der Erneuerung der Republik im Wege stand (vgl. Cic. Att. 15. 12; 16. 14; Ad Brut. 1. 15).

${ }^{54}$ Siehe Sen. Rhet. Contr. 10 praef. 5: (LABIENUS) color orationis antiquae, vigor novae; cultus inter nostrum ac prius saeculum medius, ut illum posset utraque pars sibi vindicare. libertas tanta, ut libertatis nomen excederet, et, quia passim ordines hominesque laniabat, Rabie(nu)s vocaretur. animus inter vitia ingens et ad similitudinem ingeni sui violentus et qui Pompeianos spiritus nondum in tanta pace posuisset. Vgl. z.B. Tac. Ann. 1. 2. 1 (Postquam Bruto et Cassio caesis nulla iam publica arma, Pompeius apud Siciliam oppressus exuto que Lepido interfecto Antonio ne Iulianis quidem partibus nisi Caesar dux reliquus, posito triumviri nomine consulem se ferens et ad tuendam plebem tribunicio iure contentum, ubi militem donis, populum annona, cunctos dulcedine otii pellexit, insurgere paulatim, munia senatus magistratuum legum in se trahere, nullo adversante, cum ferocissimi per acies aut proscriptione cecidissent, ceteri nobilium, quanto quis servitio promptior, opibus et honoribus extollerentur ac novis ex rebus aucti tuta et praesentia quam vetera et periculosa mallent.)

${ }^{55}$ Es ist mir klar, dass der sich anbietende Vergleich mit dem Augustusbild, das von Senecas Sohn, dem Philosophen Seneca, gegeben wird, unbeachtet blieb. Ich weise auf die bestehenden Studien hin: insb. JAL, P.: Images d'Auguste chez Sénèque. REL 35 (1957) 242-264 ; GRIMAL, P.: Sénèque et Tacite juges d'Auguste et de son époque. In BARCHIESI, A.: L'età augustea vista dai contemporanei e nel giu- 
zu verfügen und seine möglichen Mitteilungen „zwischen den Zeilen“ mit seiner Schilderung der Geschichte vergleichen zu können. Wohl oder übel bleiben uns nur Annahmen und Vermutungen.

Katarina Petrovićová

Dept. of Classical Studies

Masaryk University Brno

dizio dei posteri: atti del convegno (Mantova, Palazzo Ducale, 21-22-23 maggio 1987). Mantova 1988, $155-172$. 